\title{
Role of Cytological Grading in Prognostication of Breast Carcinoma
}

\author{
Smita, Surendra. Masamatti ${ }^{1}$, Vijaya $C^{1 *}$ and Narayana Swamy DM $^{2}$ \\ ${ }^{1}$ Department of pathology, Sapthagiri institute of medical sciences and research centre, Bangalore India \\ ${ }^{2}$ Department of community medicine, BGS Global institute of medical sciences, Bangalore, India
}

\begin{abstract}
Background: Breast carcinoma is now one of the most common cause of death in females worldwide, hence should be early diagnosis and treatment is necessary. This study aims at establishing Fine needle aspiration cytology (FNAC) as an individual parameter in diagnosing and grading breast carcinoma followed by correlation with histological grading.
\end{abstract}

Methods: Cytological smears of 51 cases of breast carcinomas were graded by using Robinson's method and corresponding histology sections were graded according to Elston and Ellis Nottingham modification of Bloom Richardson's method and correlation was done.

Result: On cytology, there were 10,26 and 15 cases of grade I, grade II and grade III tumours respectively while on histology there were 12 , 31 and 8 cases of grade I, grade II and grade III tumours. The concordance rates between cytology and histology grades were $90 \%, 76.92 \%$ and $26.67 \%$ respectively with an absolute concordance rate of $64.71 \%$.

Conclusion: FNAC is simple, non invasive and a very good tool which provides information about the aggressiveness of the tumour and helps to select appropriate treatment.

\section{Keywords: Elston and Ellis's Nottingham Modification of Bloom Richardson's Method, FNAC, Histological Grading, Robinson's Cytological Grading.}

\section{Introduction}

Fine needle aspiration cytology (FNAC) is being used increasingly to determine the nature of the breast lesions and also it provides the various intrinsic features of tumour and also their prognosis. ${ }^{[1]}$ Due to lack of awareness and absence of screening program, majority of breast cancer cases are diagnosed at a relatively advanced stage. Hence FNAC becomes the primary investigation for the diagnosis of breast carcinoma. ${ }^{[2]}$

In developed countries, the utility of FNAC is overshadowed by the needle core biopsy, where as in developing countries like India, the core biopsy is still not practiced routinely. For resource poor countries, FNAC when compared to needle core biopsy, is less invasive, cheaper, can sample different areas of the lesion in same setting at no added expenses and usually the results are accurate and are obtained on the same day. Surgeons go for a radical surgery, just based on FNAC diagnosis. But unfortunately, instead of signing a more precise "surgical pathology" type of FNAC report, it is only limited to categorizing the breast lesions as benign or malignant. The various prognostic markers are important for deciding the treatment modality by the surgeon to avoid needless morbidity.
The National cancer institute (NCI) Bethesda, sponsored a conference on the "uniform approach to report breast FNACs. They recommended the tumour grading on FNA material should be incorporated in cytological report for its prognostification. Various different grading systems are used for breast cancer cytology ${ }^{[3]}$. The present study used Robinson's method for grading of breast malignancy followed by comparison with histological grading proposed by Nottingham's modification of Bloom and Richardson system by Elston and Ellis.

\section{Materials and Methods}

This is a two year study, one year retrospective and one year prospective study of breast carcinomas diagnosed on FNAC from the period, July 2015 to August 2017 carried out in the department of pathology, Sapthagiri Institute of medical sciences, Bangalore, India. After obtaining clearance from the institutional ethical committee, all records diagnosed as breast carcinoma cases were retrieved from the cytopathology section.

Inclusion criteria: Fifty one cases of breast cancer diagnosed on FNAC and confirmed on histopathology were included in the study. 
Exclusion criteria: Cases diagnosed on FNAC but not confirmed on histopathology.

Retrospective study material comprised of 30 cases and prospective 21 cases.

FNAC was done by using $10 \mathrm{ml}$ syringe with $22-23$ gauge needle using aseptic precautions. Smears were air dried and stained with leishman stain and others were alcohol fixed and stained with papanicolau and H \& E stain. Cytological features were carefully observed and breast carcinoma was graded using Robinson's method by two independent observers.

Robinson's grading system included the following criteria: Cell dissociation (clusters/single cells),Cell size (1-2/3-4/>5xRBC size), Cell uniformity (monomorphic/ mildly pleomorphic/ pleomorphic), nucleoli(indistitinct/ noticeable/prominent), nuclear chromatin (vesicular/ granular/clumped or cleaved) and nuclear margins (smooth/folds/buds or tufts).Each of the above criteria was given scores 1-3 and total sum of scores of all criteria were used to grade the tumours. Based on this, carcinoma breast cases were graded into grade I (score 6-11), grade II (score 12-14) and grade III (score 15-18) respectively. ${ }^{[1]}$

Surgical specimens received for histopathological examination were fixed in $10 \%$ formalin. Three to four sections of around $5 \mu \mathrm{m}$ thickness were taken from the tumour and stained with $\mathrm{H} \& \mathrm{E}$. Histological grading was done according to Elston and Ellis Modification of Bloom and Richardson's method. Criteria such as tubule formation, nuclear pleomorphism and mitotic figures were evaluated. Cytological and histological grading was correlated and analysis was done.

Statistical analysis: All recorded data were entered using MS Excel software and analyzed using MEDCALC and SPSS 22 version software for determining the statistical significance. Results were expressed as mean, standard deviation and proportions. The Fisher's exact test and Chi square test was used to determine whether there was a statistical difference between the grading. "P" value of $>0.05$ was considered not to be statistically significant, $<0.05$ was considered to be statistically significant, a value of $<0.01$ was highly statistically significant and a value of $<0.001$ was considered as extremely statistically significant. Sample size $=51$.

\section{Result}

In our study, all cases of carcinoma breast were seen in females (100\%). Among 51 cases, a higher proportion of the patients were in the age group of $41-50(33.33 \%)$, the youngest patient being 27 years old and oldest patient was 82 years old. The Robinson's cytological grading which was employed had a diagnostic accuracy of $92.16 \%$, sensitivity of $75 \%$ and specificity of $97.44 \%$. The disease prevalence in our study was $23.53 \%$.

Robinson's grading system was compared with that of histological grading showed $19.6 \%$ as grade I, while $23.5 \%$ were grade I by histological grading. A higher proportion $60.8 \%$ of tumors were categorized as grade II by histological grading as compared with $51 \%$ by Robinson's grading system. And $15.7 \%$ as grade III by histological grading method while it was $29.4 \%$ by Robinson's method. So the p-value $(<0.05)$ suggests strong association between Robinson's and histological grading systems (Table 1).

The concordance rate for each grade obtained by comparing between cytological grade and histological grade obtained in our study was $90 \%, 76.92 \%$ and $26.67 \%$ for grade I, grade II and grade III respectively (Table 2). However the overall absolute concordance rate was $64.71 \%$, which was found to be fairly comparable to that reported by previous studies (Table 3 ).

The present study showed majority of cases around 28 cases i.e. $54.90 \%$ positive for axillary lymphnode metastasis and all were ipsilateral lymphnodes.

Table 1: Comparison of Robinson's grading with histological grading.

\begin{tabular}{|c|c|c|c|c|c|}
\hline \multirow{2}{*}{$\begin{array}{c}\text { Robinson's } \\
\text { grading }\end{array}$} & \multicolumn{3}{|c|}{ Histological grading } & \multirow{2}{*}{ Total } & \multirow{2}{*}{ Percentage (\%) } \\
\cline { 2 - 5 } & I & II & III & & 19.6 \\
\hline I & 9 & 1 & 0 & 10 & 51.0 \\
\hline II & 2 & 20 & 4 & 26 & 29.4 \\
\hline TII & 1 & 10 & 4 & 15 & 100 \\
\hline$\%$ & 12 & 31 & 8 & 51 & \\
\hline
\end{tabular}


Table 2: Comparison of concordance rates between the Robinson's and histological grades.

\begin{tabular}{|c|c|c|c|}
\hline Grade & $\begin{array}{c}\text { No of concordant cases between } \\
\text { Robinson's and histological grades }\end{array}$ & $\begin{array}{c}\text { No of cases in each } \\
\text { Robinson's grade }\end{array}$ & Concordance rate (\%) \\
\hline I & 9 & 10 & 90.00 \\
\hline II & 20 & 26 & 76.92 \\
\hline III & 4 & 15 & 26.67 \\
\hline Total & $\mathbf{3 3}$ & $\mathbf{5 1}$ & Absolute concordance rate: $\mathbf{6 4 . 7 1}$ \\
\hline
\end{tabular}

Table 3: Comparison of concordance rates in different studies with the present study employing Robinson's cytological grading system.

\begin{tabular}{|c|c|}
\hline Studies & Concordance rate (\%) \\
\hline Das et a $^{[[]]}$ & 71.2 \\
\hline Chhabra et al[5] $^{[[5]}$ & 65 \\
\hline Sultana and Rehman $^{[6]}$ & 86.3 \\
\hline Khan et al $^{[[]]}$ & 89.1 \\
\hline Sinha et al & 69.5 \\
\hline Lingegowda et al[9] $^{[8]}$ & 64 \\
\hline Present study & 64.71 \\
\hline
\end{tabular}

\section{Discussion}

FNAC is generally considered as a rapid, reliable and safe diagnostic tool to diagnose both nonneoplastic and neoplastic breast lesions. It has a sensitivity ranging from $76 \%$ to $99 \%$ and specificity ranging from $60 \%$ to $100 \%$. ${ }^{[10]}$

Grading of tumours before surgery is very important as appropriate medical regimen can be initiated. ${ }^{[11]}$ Nuclear grading is considered as one of the most important prognostic factor in carcinoma breast and also it is very well correlated with histological sections when compared with other parameters. ${ }^{[12]}$ The interobserver and intraobserver observations are the key factors in any grading systems. ${ }^{[13]}$

Several cytological gradings have been proposed in the past, such as, Taniguchi's, Fisher's, Mouriquand's, Robinson's, Howell's etc. Among all Robinson's cytological grading is easy and reproducible for evaluation and prognostification of all breast lesions without any additional morbidity or expenses to the patient. ${ }^{[10]}$ The aim of cytoprognostic grading is diagnose the fast growing tumours as they are more likely to respond to chemotherapy. ${ }^{[5]}$

The optimal number of cell clusters needed to define the adequacy on FNA smears is not clear, although many authors have used six as the definite number of cell clusters. Singh et al showed that a better concordance between these grading systems can be achieved by examining 8 cell clusters. ${ }^{[14]}$

Multiple regression analysis of various cytological features was used to assess the significance of each cytological parameter in the past. The coefficient of regression for all the parameters used in Robinson's grading were statistically significant $(\mathrm{p}=0.000)$ and all of them equally contributed in determining the cytological grading of the tumour. ${ }^{[15]}$

In the present study, Robinson's grading corresponds well with the histopathological grading. Majority of the tumours were grade II (51\%) followed by grade III (29.4\%) and grade I (19.6\%). Many authors such as Robinson et al, Meena et al, ${ }^{[16]}$ Chandanwale shirish $\mathrm{S}$ et al, ${ }^{[17]}$ Taniguichi et al ${ }^{[18]}$ had similar findings and is in concurrence with our study, whereas studies done by Vidya Vasudev et al ${ }^{[19]}$ found majority of tumours in grade III followed by grade II, while Pandya AN et al ${ }^{[2]}$ and Neelam Sood et $\mathrm{al}^{[20]}$ found majority of the tumours in grade I, followed by grade II.

On histological grading, $60.8 \%$ of cases belong to grade II, $23.5 \%$ cases belong to grade I and $15.7 \%$ cases to grade III. This shows cytological grading was very well correlated with the histological grading with a concordance rate of $64.71 \%$, which is again comparable with studies done by Lingegowda et al. ${ }^{[9]}$

In our study, the concordance of Robinson's cytological grading with histological grading was $90 \%$ for grade I tumours, $76.92 \%$ for grade II tumours and $26.67 \%$ for grade III tumours respectively. The overall concordance rate $64.71 \%$ which is comparable with other reported studies like Lingegowda et al, Robinson's et al. However Dinisha Einstein et al ${ }^{[21]}$ in 2014 gave a concordance rate of $77.7 \%$ by comparing 7 cytological grades.

The present study showed, lower concordance rates were observed in grade III tumours, which may be due to 
subjectivity among the investigators and small sample size. In cytological grading, importance was given to nuclear features like nuclear membrane, nuclear size, nucleoli, pattern of the chromatin as compared to histological grading where nuclear feature is only one component. And also identification of mitosis and tubule formation on FNA smears was difficult as observed by Howell et al, ${ }^{[22]}$ Dinisha Einstein et al. ${ }^{[21]}$ This may be due to the fact that the cytological material aspirated is very less as compared with the histological sections. Other factors responsible for lower concordance rate include poor quality slides with fixation artifacts, severe inflammation and tumour necrosis.

Our study showed majority (54.90\%) of the patients had lymph node metastasis, and all were ipsilateral lymphnodes. Similar findings were reported by Vidya Vasudev et al ${ }^{[19]}$ and Navita gupta et al. ${ }^{[15]}$

Limitations of the study: 1) Small study sample 2) Difficulty in identifying mitosis and tubules on cytology aspirates 3) Interobserver variability 4) Cannot distinguish between DCIS (Ductal carcinoma in situ) and invasive breast carcinoma.

\section{Conclusion}

Our study showed that Robinson's grading system of carcinoma breast is easy, quick and correlated well with histological grading. Cytological grading may be used as substitute for histological grading and FNAC in combination with mammography can provide information regarding the tumour type, size and grading before surgery which will help to choose the appropriate neoadjuvant therapy and prevents unnecessary over treatment of low grade carcinomas.

\section{Acknowledgements}

To Mr Narayanaswamy DM, Assistant professor and statistician, Department of community medicine, BGS Global institute of medical sciences , Bangalore, for statistical analysis of the data.

\section{Reference}

1. Wani FA, Bhardwaj S, Kumar D, Katoch P. Cytological grading of breast cancers and comparative evaluation of two grading systems. J Cytol 2010; 27(2): 55-58.

2. Pandya AN, Shah NP. Comparative Evaluation of Robinson's Cytological Grading with Elston and Ellis' Nottingham Modification of Bloom Richardson Histopathology Grading for Breast Carcinoma. Natl J Community Med 2012; 3(3):491-5.

3. Bansal C, Pujani M, Sharma KL, Srivastava AN, Singh US. Grading systems in the cytological diagnosis of breast cancer: A review. J Can Res Ther 2014; 10:839-45.
4. Das AK, Kapila K, Dinda AK, Verma K. Comparative evaluation of grading of breast carcinomas in fine needle aspirates by two methods. Indian J Med Res 2003; 247-250.

5. Chhabra S, Singh PK, Agarwal A, Bhagoliwal A, Singh SN. Cytological grading of breast carcinoma: A multivariate regression analysis. J Cytol 2005; 22:62-5.

6. Sultana T, Rahman AJ. Cytological grading of duct cell carcinoma, NOS of breast and its correlation with histological grading. Bangladesh Med Res Counc Bull.2006; 32(2):49-54.

7. Khan MZ, Haleem A, AI Hassani H, Kfoury H. Cytopathological grading, as a predictor of histopathological grade, in ductal carcinoma (NOS) of breast, on air-dried Diff-Quik smears. Dign Cytopathol 2003; 29:185-93.

8. Sinha SK, Sinha N, Bandyopadhyay R, Mondal SK. Robinson's cytological grading on aspirates of breast carcinoma: Correlation with Bloom Richardson's histological grading. J Cytol 2009; 26:140-3.

9. Lingegowda JB, MuddeGowda PH, Ramakantha CK, Chandrasekar HR. Cytohistological correlation of grading in breast carcinoma. Diagn Cytopathol 2011; 39:251-7.

10. Arul P, Masilamani S. Comparative evaluation of various cytomorphological grading systems in breast carcinoma. Indian J Med Paediatr Oncol 2016; 37:79-84.

11. Robinson IA, McKee G, Kissin MW. Typing and grading of breast carcinoma on fine-needle aspiration: is this clinically useful information? Diagn Cytopathol 1995; 13: 260-5.

12. Zoppi JA, Pellicer EM, Sundblad AS. Cytohistologic correlation of nuclear grade in breast carcinoma. Acta Cytol 1997; 41:701-4.

13. Cajulis RS, Hessel RG, Hwang S, Haines K, FriasHidvegi D, O'Gorman M. Simplified nuclear grading of fine-needle aspirates of breast carcinoma: concordance with corresponding histologic nuclear grading and flow cytometric data. Diagn Cytopathol 1994; 11:124-30.

14. Mustaphi RM, Chowdhury S, Mondal S, Bhattacharya SK, Sarkar S, Saha Tk. Role of cytological grading in breast cancer prognosis and its histopathological correlation. Journal of dental and medical sciences 2014;13(10):106109.

15. Gupta N, Mangal K. Mehra M. "Diagnostic accuracy of Robinson's cytological grading assuming modified Scarff bloom Richardson's histological grading as a gold standard in malignant Neoplasms of breast. International journal of Current Research 2016; 8 (01):25429-25435.

16. Meena SP, Hemrajani DK, Joshi N. A comparativeand evaluative study of Cytological and Histological grading system profile in malignant neoplasm of breast-an important prognostic factor. Indian J Pathol Microbiol 2006; 49(2):199202.

17. Chandanwale SS, Mishra N, Kaur S, Paranjape S, Pandey A, Jha M. Comparative analysis of six cytological grading systems in breast carcinoma. Clin Cancer Investig J 2016; 5:409-15. 
18. Taniguchi E, Yang Q, Tang W, Nakamura Y, Shan L, Nakamura M, et al. Cytological grading of invasive breast carcinoma. Correlation with clinicopathologic variables and predictive value of nodal metastasis. Acta Cytol 2000;44:587-91.

19. Vasudev, Vidya, Rangaswamy R., Geethamani V. "The Cytological Grading of Malignant Neoplasms of the Breast and Its Correlation with The Histological Grading." Journal of Clinical and Diagnostic Research 2013; 7(6): 1035-1039.

20. Sood N, Nigam JS, Yadav P et al. Comparative study of cytomorphological Robinson's grading of breast carcinoma with Modiied Bloom-Richardson histopathological grading. Pathology research international 2013; 146542:1-5.

21. Einstien D, Omprakash BO, Ganapathy H, Rahman S. Comparison of 3-tier cytological grading systems for breast carcinoma. ISRN Oncol 2014; 2014:252103.

22. Howell LP, Gandour-Edwards R, O'Sullivan D. Application of the Scarff-Bloom-Richardson tumor grading system to fine-needle aspirates of the breast. Am J Clin Pathol 1994;101:262-5.

*Corresponding author:

Dr Vijaya C, Professor and head of the department, Department of pathology, 3rd floor, Sapthagiri medical college, Bangalore 560090 India Phone: +91 9741147555

Email: vijayachowdappa18@gmail.com

Financial or other Competing Interests: None. 